\title{
A rare case of a splenic hamartoma in a patient with a huge palpable abdominal mass: a case report
}

\author{
Paraskevi Vlachou', Dimitris Fagkrezos²*, Anastasia Tzivelopoulou', Georgia Kyriakopoulou', Petros Maniatis², \\ Charikleia Triantopoulou' ${ }^{1}$ and John Papailiou ${ }^{2}$
}

\begin{abstract}
Introduction: Splenic hamartoma is a primary benign tumor of the spleen, which is often found incidentally. Splenic hamartomas are very rare, with approximately 150 cases documented in the literature to date. They represent benign vascular proliferation. Histological findings consist of disorganized stroma and vascular channels of varying width, with or without lymphoid follicles.

Case presentation: We present the case of a 39-year-old Greek woman, with no significant medical history, who was diagnosed incidentally with an enormous splenic hamartoma on computed tomography, finally confirmed by surgery and histopathology. Hamartomas are benign lesions, and it is important to differentiate them from malignancy.

Conclusion: Hamartoma represents a rare vascular entity characterized by a cluster of differentiation 8-positive immunophenotype. It is usually asymptomatic but large hamartomas may present with symptoms such as hemopoetic disorders, which resolve after splenectomy. It is important for radiologists to be able to differentiate splenic hamartoma from malignant entities.
\end{abstract}

Keywords: CT, Hamartoma, MRI findings, Spleen, Splenic hemangioma, Splenoma

\section{Introduction}

In terms of structure and function, the spleen consists of white and red pulp [1]. White pulp plays an important role in the immune system by producing lymphocytes, plasma cells and antibodies. Red pulp filters blood, by removing microorganisms, old red cells and several antigens. In young people, the spleen also has a hemopoetic role. The length of the spleen does not exceed $13 \mathrm{~cm}$ in men and $12 \mathrm{~cm}$ in women. Its weight is between $80 \mathrm{~g}$ and $300 \mathrm{~g}$, with a median weight of about $150 \mathrm{~g}$.

Vascular neoplasms are the most common primary neoplasms of the spleen. Spleen hamartoma was described first in 1861 by Rokitansky [2], and has also been called splenoma or spleen within a spleen.

\footnotetext{
* Correspondence: fdbl@ath.forthnet.gr

${ }^{2}$ Computed Tomography Department, Konstantopouleio General Hospital, Agias Olgas 3-5, 14233 Nea Ionia, Greece

Full list of author information is available at the end of the article
}

Benign splenic tumor-like masses are generally rare, with an incidence of seven cases in 100,000 autopsy specimens, and most are cysts or hemangiomas. Hamartomas are extremely rare, with an incidence of three cases in 200,000 splenectomies [3].

With the rapid improvement of imaging modalities, the detection of small and asymptomatic lesions is now easy, and a differential diagnosis can be achieved in a timely manner with high accuracy.

\section{Case presentation}

A 39-year-old Greek woman, with no remarkable medical history, presented to the emergency room of our hospital with diffuse abdominal pain and a mass-like distention of the left side of her abdomen. No weight loss was reported.

On physical examination we found a big painless, palpable mass on the left side of her abdomen. She had 
normal vital signs and laboratory findings were normal, except for mild leukocytosis.

An abdominal ultrasound showed a huge, mostly hypoechoic, mass that was impossible to characterize. Its origin was also difficult to define.

Abdominal computed tomography (CT) was performed, and images before the administration of contrast material showed a slightly hypodense, well-circumscribed, encapsulated mass on her left abdominal side, measuring $23 \times 17 \times 23 \mathrm{~cm}$ (Figure 1 ). This mass was compressing other abdominal organs to the right, with no obvious invasion. After contrast bolus injection, in the arterial phase, we noted heterogenous enhancement, with higher peripheral contrast media concentration (Figure 2). No vessel invasion was noticed. In the late venous phase, the mass became isodense. No cystic areas were observed but microcalcifications were noted (Figure 3).

Magnetic resonance imaging (MRI) showed a high signal intensity mass on T2-weighted images, presenting intense gadolinium enhancement (Figures 4 and 5). The possibility of splenic hamartoma was proposed. This was confirmed after surgical removal of her spleen and a biopsy of the mass.

Histological examination revealed disorganized red pulp-like stroma with lipocytes and disorganized vessels of variable width, lined by slightly plump endothelial cells without atypia, with or without white pulp (Figure 6).

After surgical intervention, additional CT imaging was performed as a follow-up. This showed normal post-surgical evolution, with her other organs in normal positions and only some post-surgical lesions, such as adipose tissue thickening and fibrous remnants (Figure 7).

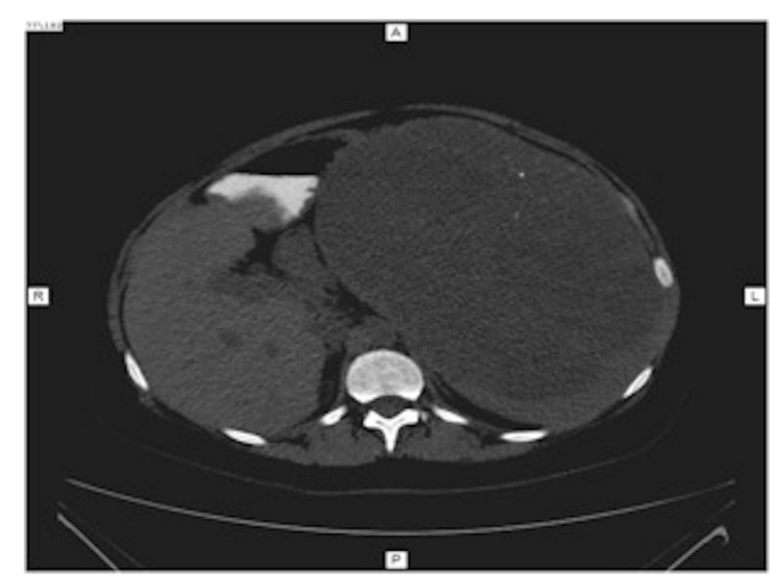

Figure 1 Computed tomography imaging of splenic hamartoma before contrast injection. Note the presence of two microcalcifications.

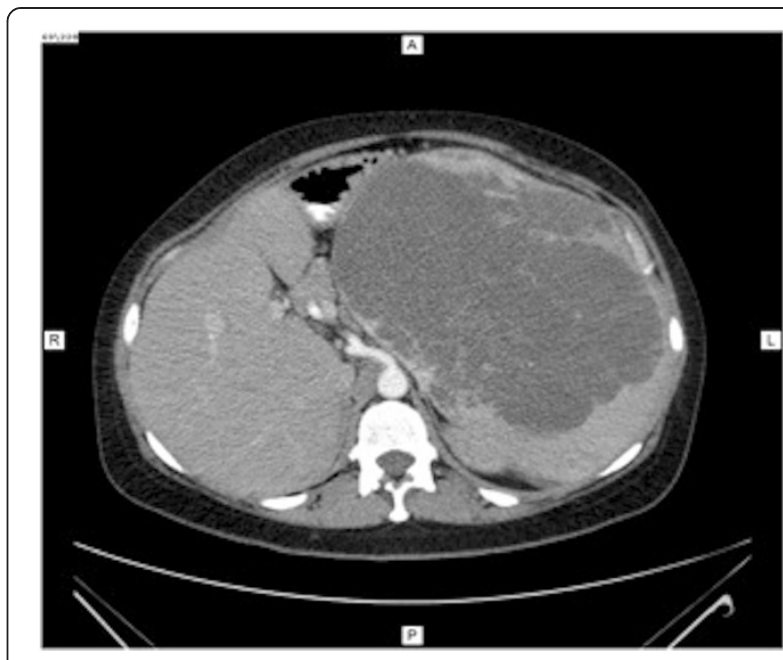

Figure 2 Computed tomography imaging of the splenic mass in arterial phase.

\section{Discussion}

The pathogenesis of hamartomas is controversial. Some consider hamartomas to be a congenital malformation of splenic red pulp, others a neoplasm or reaction to trauma $[1,4]$. The spleen is an uncommon place for primary tumor origin, so tumors located here are most commonly vascular (both benign and malignant).

Splenic hamartomas must be differentiated from other vascular tumors of the spleen depending on the cell of origin, such as hemangioma, littoral cell angioma, lymphangioma, hemangioendothelioma or angiosarcoma [4]. Solid mass-forming lesions, such as metastases and lymphoma, mycobacterial infections, and sarcoidosis must be included in the radiological differential diagnosis [5].

Hemangioma is the most common benign tumor of the spleen, arising from endothelial cells of the sinusoids

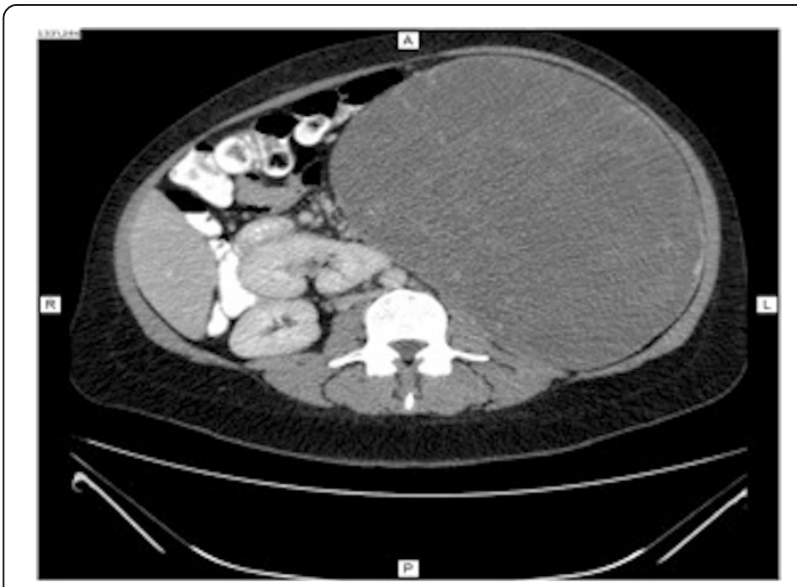

Figure 3 Computed tomography imaging of the splenic mass in venous phase. 


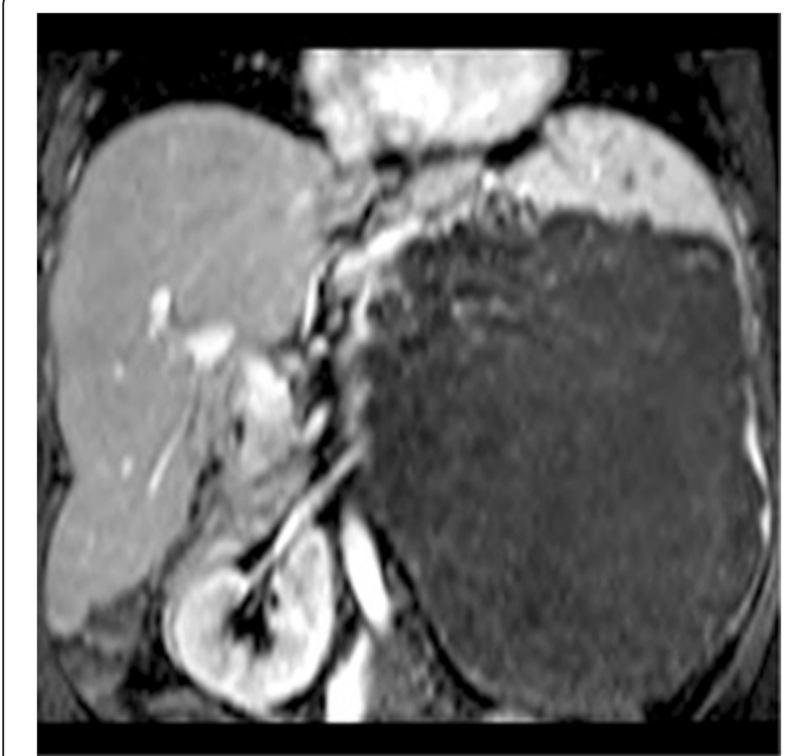

Figure 4 Coronal T1-weighted plus gadolinium magnetic resonance imaging of splenic hamartoma.

and usually measuring less than $2 \mathrm{~cm}$ [6]. Littoral cell angioma is a rare vascular tumor, arising from littoral cells of the splenic sinuses $[4,7,8]$. This rare lesion was first described in 1991 and histological findings demonstrate anastomosing vascular channels lined by cells positive to both endothelial and histiocytic markers. Lymphangioma is another rare benign spleen tumor, which manifests as a subcapsular nodule or as diffuse lymphangiomatosis in young patients. Hemangioendothelioma is a quite rare and controversial entity of the spleen, with an intermediate histology between that of a hemangioma and an angiosarcoma, with lining cells showing an intermediate degree of atypia. Finally, angiosarcoma is a malignant primary

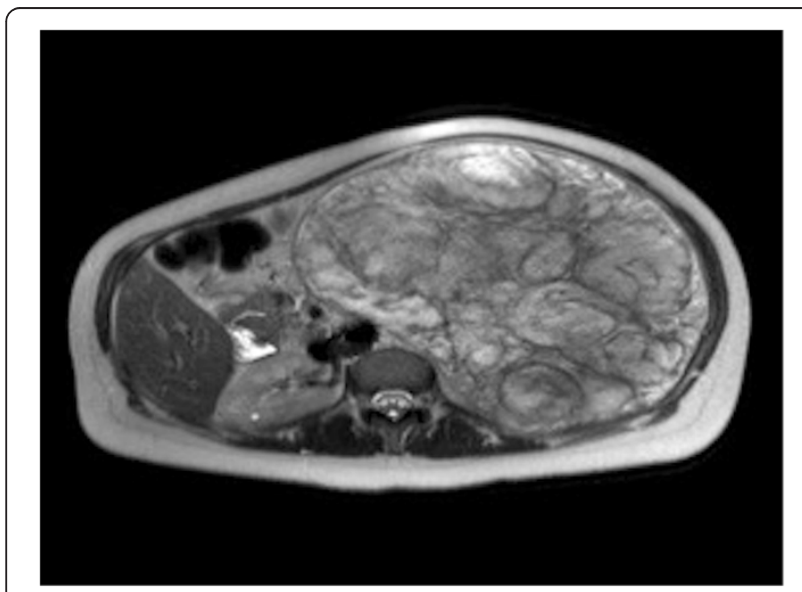

Figure 5 Axial T2-weighted magnetic resonance imaging of splenic hamartoma.

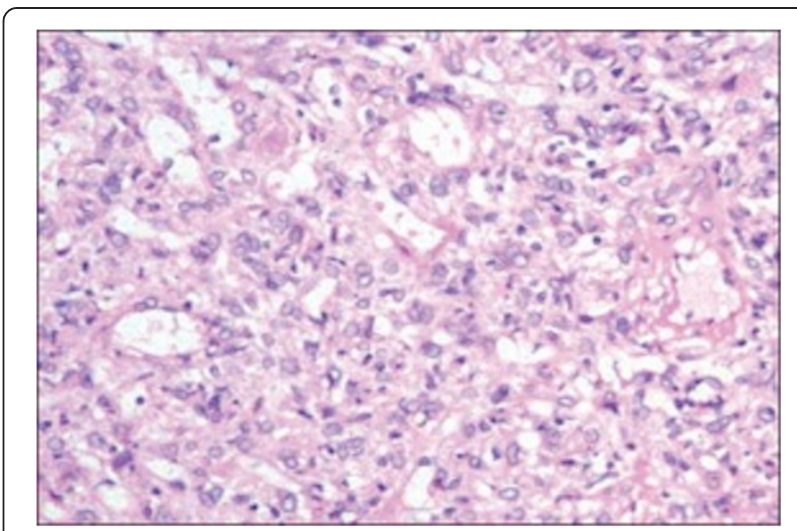

Figure 6 Histological examination revealed disorganized stroma and vessels, with endothelial cells without atypia.

tumor of non-lymphoid origin. Irregular, anastomosing vessels, cellular atypia and invasion of adjacent organs can lead to a definitive diagnosis.

Hamartomas are quite rare lesions (about three cases in 200,000 splenectomies), which occur in any age group [1]. They seem to occur equally in male and female patients, but women seem to have larger lesions, probably due to hormonal influence. The tumor size ranges from a few millimeters up to $20 \mathrm{~cm}$. In our patient, the impressive finding was the enormous size $(23 \times 17 \times 23 \mathrm{~cm})$ of the lesion, reaching almost the entrance of her pelvis.

Generally, hamartomas are asymptomatic. Only large lesions are able to manifest clinical symptoms, such as palpable mass, spontaneous rupture with intra-abdominal bleeding, and hypersplenism (thrombocytopenia, anemia, pancytopenia) $[5,6,9]$. This clinical symptomatology seems to occur more often in women. Most cases occur in adult patients.

Diagnosis is enhanced by imaging findings. Recently, Wang et al. [5] described the radiological features of

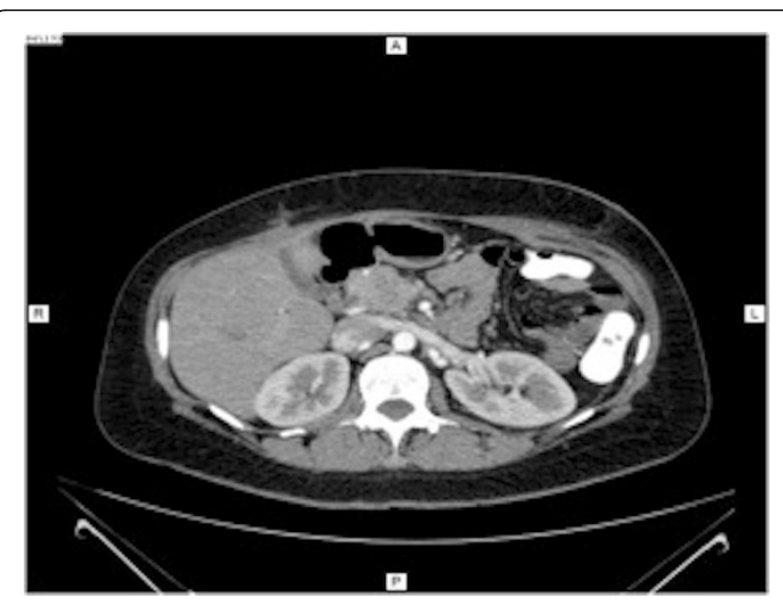

Figure 7 Computed tomography imaging after surgical resection of huge splenic hamartoma. 
hamartomas. Ultrasound findings usually include a hyperechoic mass with or without cystic areas or calcifications. Color Doppler usually shows a hypervascular mass. On CT, a hamartoma appears as a solid single or, less often, multiple masses that are well-circumscribed and encapsulated. The mass is slightly hypodense before contrast administration. After contrast injection, prolonged enhancement is achieved in a single mass, although low-density masses are observed in multiple splenic hamartomas. MRI distinguishes fibrous from non-fibrous hamartomas. MRI findings include an isointense mass in T1-weighted images and hyperintense mass in T2weighted images $[4,8,10,11]$.

Finally, diagnosis is confirmed by histopathological examination and is enforced by immunohistopathology, with a splenic hamartoma having a cluster of differentiation 8 (CD8)-positive immunophenotype [4].

\section{Conclusion}

Hamartoma represents a rare vascular proliferation characterized by CD8-positive immunophenotype. It is usually asymptomatic, but large tumors may present with symptoms such as hemopoetic disorders, which resolve after splenectomy. It is important for radiologists to be able to differentiate splenic hamartoma from malignant entities.

\section{Consent}

Written informed consent was obtained from the patient for publication of this case report and accompanying images. A copy of the written consent is available for review by the Editor-in-Chief of this journal.

\section{Abbreviations}

$\mathrm{CT}$ : computerized tomography; MRI: magnetic resonance imaging

\section{Competing interests}

The authors declare that they have no competing interests.

\section{Authors' contributions}

DF analyzed and interpreted the patient data regarding the differential diagnosis of the disease, interpreted the radiologic findings, and was a major contributor in writing the manuscript. PV interpreted the radiologic examination findings and was a major contributor in writing the manuscript. AT interpreted the radiologic examination findings. GK interpreted the radiologic examination findings and $\mathrm{PM}$ analyzed and interpreted the radiologic examination findings. $C T$ designed and reviewed the manuscript. JP designed and reviewed the manuscript. All authors read and approved the final manuscript.

\section{Author details}

'Department of Radiology, Konstantopouleio General Hospital, Agias Olgas 3-5, 14233 Nea lonia, Greece. ${ }^{2}$ Computed Tomography Department, Konstantopouleio General Hospital, Agias Olgas 3-5, 14233 Nea Ionia, Greece.

Received: 22 June 2014 Accepted: 18 November 2014 Published: 28 January 2015

\section{References}

1. Silverman ML, LiVolsi VA. Splenic hamartoma. Am J Clin Pathol. 1978;70:224-9.
2. Tsitouridis I, Michailides M, Tsitouridis K, Davidis I, Efstathiou I. Symptomatic splenoma (hamartoma) of the spleen: a case report. Hippokratia. 2010;14:54-6.

3. Lam KY, Yip KH, Peh WC. Splenic vascular lesions: unusual features and a review of the literature. Aust N Z J Surg. 1999;69:422-5.

4. Lee H, Maeda K. Hamartoma of the spleen. Arch Pathol Lab Med. 2009;133:147-51

5. Wang JH, Ma XL, Ren FY, Zuo CJ, Tian JM, Wang ZF, et al. Multi-modality imaging findings of splenic hamartoma: a report of nine cases and review of the literature. Abdom Imaging. 2013;38:154-62.

6. Kaza RK, Azar S, Al-Hawary MM, Francis IR. Primary and secondary neoplasms of spleen. Cancer Imaging. 2010;10:173-82.

7. Sim J, Han H, Jin Jun Y, Rehman A, Jang SM. Splenic hamartoma: a case report and a review of the literature. World J Clin Cases. 2013;1(7):217-9.

8. Namikawa T, Kitagawa H, Iwabu J, Matsumoto M, Hanazaki K. Laparoscopic splenectomy for splenic hamartoma. Case management and clinical consequences. World J Gastrointest Surg. 2010;2(4):147-51.

9. Yu RS, Zhang SZ, Hua JM. Imaging findings of splenic hamartoma. World J Gastrointest Surg. 2004;10:2613-5.

10. Dachman A. Radiology of the Spleen. St Louis, MO: Mosby; 1993. p. 56-67L.

11. Kamaya A, Desser TSWS. Multiple lesions of the spleen: differential diagnosis of cystic and solid lesions. Semin Ultrasound CT MR. 2006;27:389-403.

doi:10.1186/1752-1947-9-4

Cite this article as: Vlachou et al:: A rare case of a splenic hamartoma in a patient with a huge palpable abdominal mass: a case report. Journal of Medical Case Reports 2015 9:4.

\section{Submit your next manuscript to BioMed Central and take full advantage of:}

- Convenient online submission

- Thorough peer review

- No space constraints or color figure charges

- Immediate publication on acceptance

- Inclusion in PubMed, CAS, Scopus and Google Scholar

- Research which is freely available for redistribution 